\title{
Curcumin-carrying nanoparticles prevent ischemia-reperfusion injury in human renal cells
}

\author{
Yong $\mathrm{Xu}^{1, *}$, Ning $\mathrm{Hu}^{2,}{ }^{2}$, Wei Jiang ${ }^{1, *}$, Hong-Fang Yuan ${ }^{3}$, Dong-Hui Zheng ${ }^{1}$ \\ ${ }^{1}$ Department of Nephrology, The Affiliated Huai'an Hospital of Xuzhou Medical University and The Second People's Hospital \\ of Huai'an, Huai'an 223002, China \\ ${ }^{2}$ Department of Nephrology, The First People's Hospital of Jingmen, Jingmen, Hubei 448000, China \\ ${ }^{3}$ Family Planning Research Institute, Tongji Medical College, Huazhong University of Science and Technology, Wuhan 430030, \\ China \\ * Co-first authors \\ Correspondence to: Dong-Hui Zheng, email: zddwjj@126.com \\ Hong-Fang Yuan, email: D201578150@hust.edu.cn \\ Keywords: Curcumin, nanoparticles, ischemia-reperfusion injury
}

Received: August 24, 2016

Accepted: November 08, 2016

Published: November 25, 2016

\section{ABSTRACT}

Renal ischemia-reperfusion injury (IRI) is a major complication in clinical practice. However, despite its frequency, effective preventive/treatment strategies for this condition are scarce. Curcumin possesses antioxidant properties and is a promising potential protective agent against renal IRI, but its poor water solubility restricts its application. In this study, we constructed curcumin-carrying distearoy Iphosphatidylethanolamine-polyethylene glycol nanoparticles (Cur-NPs), and their effect on HK-2 cells exposed to IRI was examined in vitro. Curcumin encapsulated in NPs demonstrated improved water solubility and slowed release. Compared with the IRI and Curcumin groups, Cur-NP groups displayed significantly improved cell viability, downregulated protein expression levels of caspase-3 and Bax, upregulated expression of $\mathrm{Bcl}-2$ protein, increased antioxidant superoxide dismutase level, and reduced apoptotic rate, reactive oxygen species level, and malondialdehyde content. Results clearly showed that Cur-NPs demonstrated good water solubility and slow release, as well as exerted protective effects against oxidative stress in cultured HK-2 cells exposed to IRI.

\section{INTRODUCTION}

Ischemia-reperfusion injury (IRI) is the damage caused by unavailability of blood supply in an organ until blood flow and oxygenation are restored [1]. Given the specificity in structure and function of the kidney, renal IRI commonly occurs and is associated with many complex clinical conditions, such as hemorrhagic shock [2], renal transplantation [3], and acute renal failure [4]. Pathogenic studies have shown that apoptosis plays a vital role in renal IRI and is reduced by an imbalance in scavenging and generation of reactive oxygen species (ROS) [5, 6], but the related mechanism is poorly understood. To our knowledge, effective preventive/treatment strategies for this condition are scarce.

Curcumin, an active component extracted from the roots and stems of Curcuma Species, such as turmeric, radix curcumae, and rhizoma curcumae, demonstrates antioxidant properties [7, 8]. A study has further indicated that curcumin ameliorates tubular necrosis by significantly reducing the formation of noxious oxidants [9]. Although Curcumin is easily metabolized, its application has been restricted because of its poor water solubility and thus is difficultly absorbed by the body. Therefore, new dosage forms of Curcumin must be developed to increase its solubility, to enhance its pharmacological effects, and to facilitate proper route of administration.

Two comprehensive reviews have recently reported that drug delivery using nanoparticles (NPs) offers numerous advantages, such as targeted delivery, slowed release, high stability, and relatively low toxicity $[10,11]$. NPs prepared from distearoylphosphatidylethanolaminepolyethylene glycol (DSPE-PEG) display bidirectional solubility in water and in fat. PEG demonstrates good water solubility and low immunogenicity and thus can be excreted from the kidney without need for any structural 
changes [12]. DSPE displays good drug encapsulation efficiency and therefore can be used as core [13]. The bidirectional solubility of DSPE-PEG increases the encapsulation efficiency and water solubility of fat-soluble drugs.

Given the advantages of nanocarriers and the pharmaceutical features of Curcumin, Curcumin-carrying long-circulating DSPE-PEG NPs were constructed in this study. The effect of these NPs on HK-2 cells exposed to IRI was examined in vitro, and the mechanism of such effect was preliminarily investigated.

\section{RESULTS}

\section{Solubility of Curcumin and Cur-NPs}

Precipitation of Curcumin in water (Figure 1A) demonstrated that Curcumin is poorly water soluble. The water solution of blank NPs is light blue and transparent, whereas that of Cur-NPs is yellow and transparent, suggesting that encapsulation in DSPE-PEG NPs enhanced the water solubility of Curcumin (Figure 1C).

\section{Size of DSPE-PEG NPs}

The mean size of Cur-NPs was $81.93 \pm 0.5 \mathrm{~nm}$. The DSPE-PEG NPs showed a narrow size distribution as determined using a particle size analyzer (Figure 2). Additionally, Lestari [14] reported that a nanosystem can maintain long-term stability when its zeta potential is $-11.1 \mathrm{mV}$.

\section{In vitro investigation on drug release}

Figure 3 shows that the release rate of Cur-NPs was significantly slower than that of non-encapsulated Curcumin. The percentages of released non-encapsulated Curcumin and Cur-NPs from a dialysis bag for the first $5 \mathrm{~h}$ were approximately $80 \%$ and approximately $30 \%$, respectively. After $40 \mathrm{~h}$, the percentage of Cur-NPs released from the dialysis bag was approximately $60 \%$. These results indicated that Cur-NPs can be slowly released, thereby maintaining a constant concentration over a long period.

\section{Effects of Curcumin and Cur-NPs on IRI- inhibited cell proliferation}

Figure 4 shows that IRI treatment significantly inhibited the proliferation of HK-2 cells; this phenomenon was indicated by the changes in optical density (OD) as revealed by methyl thiazolyl tetrazolium (MTT) colorimetry $(p<0.05)$. By contrast, Curcumin treatment significantly alleviated the inhibitory effects of IRI on cell proliferation $(p<0.05)$, and Cur-NP treatment further alleviated the inhibitory effects of IRI on cell proliferation compared with Curcumin treatment $(p<0.05)$. These results indicated that, compared with Curcumin, Cur-NPs

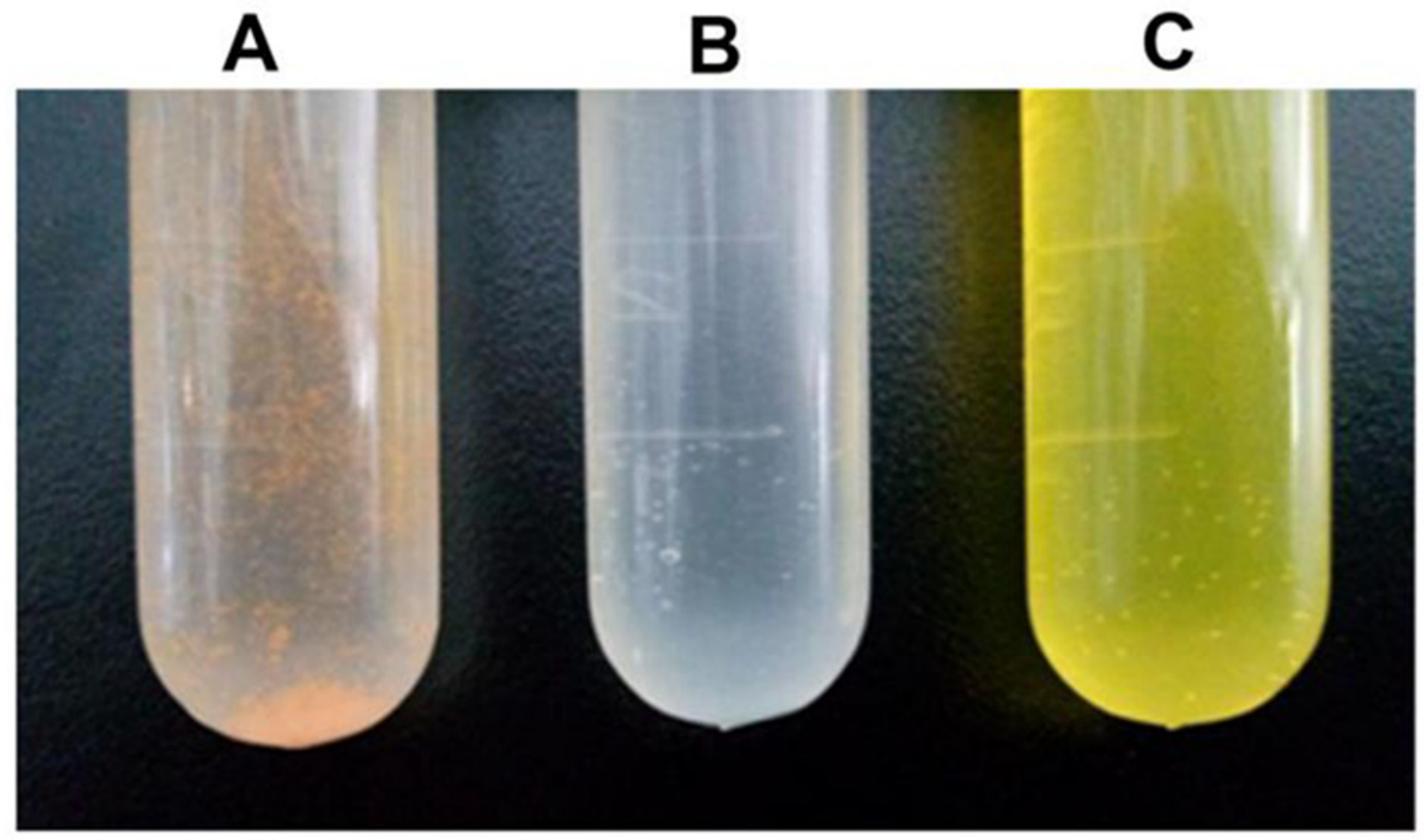

Figure 1: The solution of Cur-NPs in test tube. A. Non-encapsulated curcumin B. Blank nanoparticles C. Cur-NPs. 
exert higher protective effects against IRI injury in HK-2 cells.

\section{Effects of Curcumin and Cur-NPs on apoptosis of HK-2 cells}

Four groups of HK-2 cells were assessed. Data derived from flow cytometry profiles were qualitatively consistent with the results of replicate experiments. Treated cells were cultured for $48 \mathrm{~h}$, harvested, stained with Annexin V-FITC/PI, and then analyzed via flow cytometry.

Annexin $\mathrm{V}$ was used in flow cytometric analysis to examine the level of apoptosis in HK-2 cells. As shown in Figure 5, the Cur-NP group displayed a reduced number of apoptotic cells (15.20\% in the injury group vs. $6.20 \%$ in the Cur-NP group). These results demonstrated that the number of early and late apoptotic cells decreased in the
Curcumin group (Figure 5). The percentages of cells in the early apoptotic phase were $4.23 \%, 15.20 \%, 8.58 \%$, and $6.20 \%$ in the control, injury, Curcumin, and Cur-NP groups, respectively, and the corresponding percentages of cells in the late apoptotic phase were $2.98 \%, 4.98 \%$, $2.93 \%$, and $2.31 \%$ (Figure 5). Data suggested that CurNPs can protect HK-2 cells and that they can better alleviate apoptosis than Curcumin.

\section{Determination of intracellular reactive oxygen content by using DCFH-DA fluorescence probe}

Intracellular ROS level was detected via fluorescent staining. The IR cells clearly showed an enhanced intensity of yellow-green fluorescence, demonstrating that intracellular ROS level increased after IRI; this result indicated that oxidative stress response plays an important role in renal IRI. Fluorescence intensity decreased both in

Statistics Graph (1 measurements)

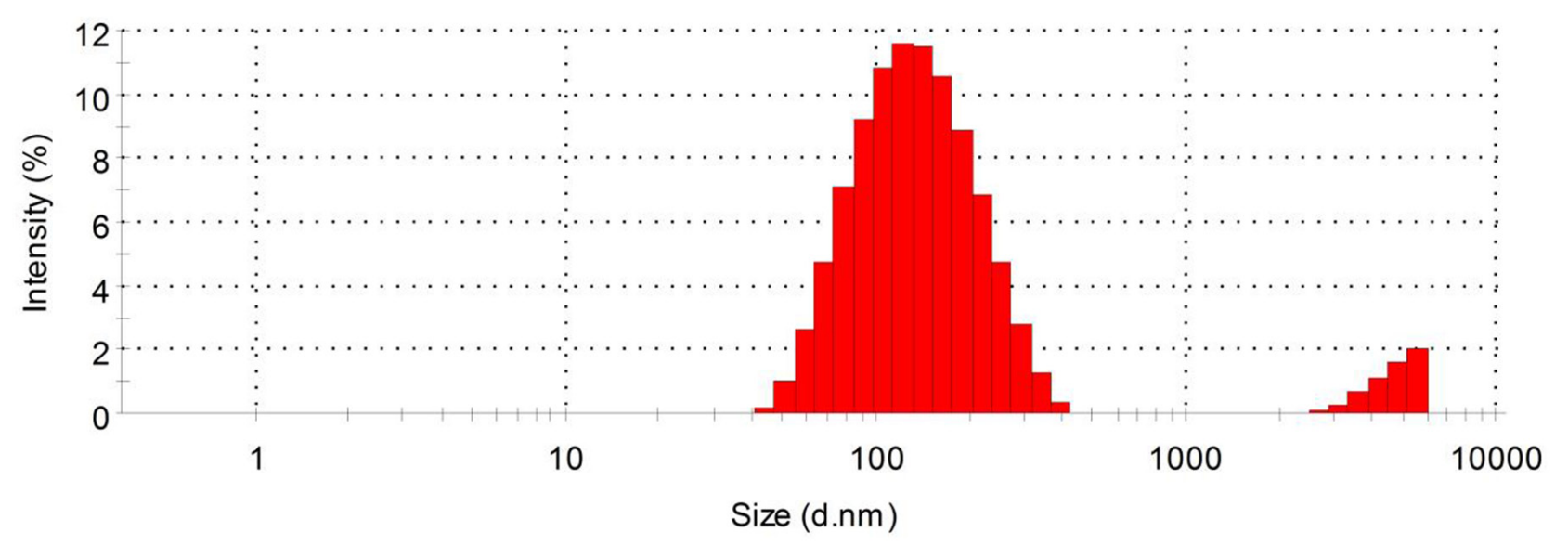

Size Dis tribution by Number

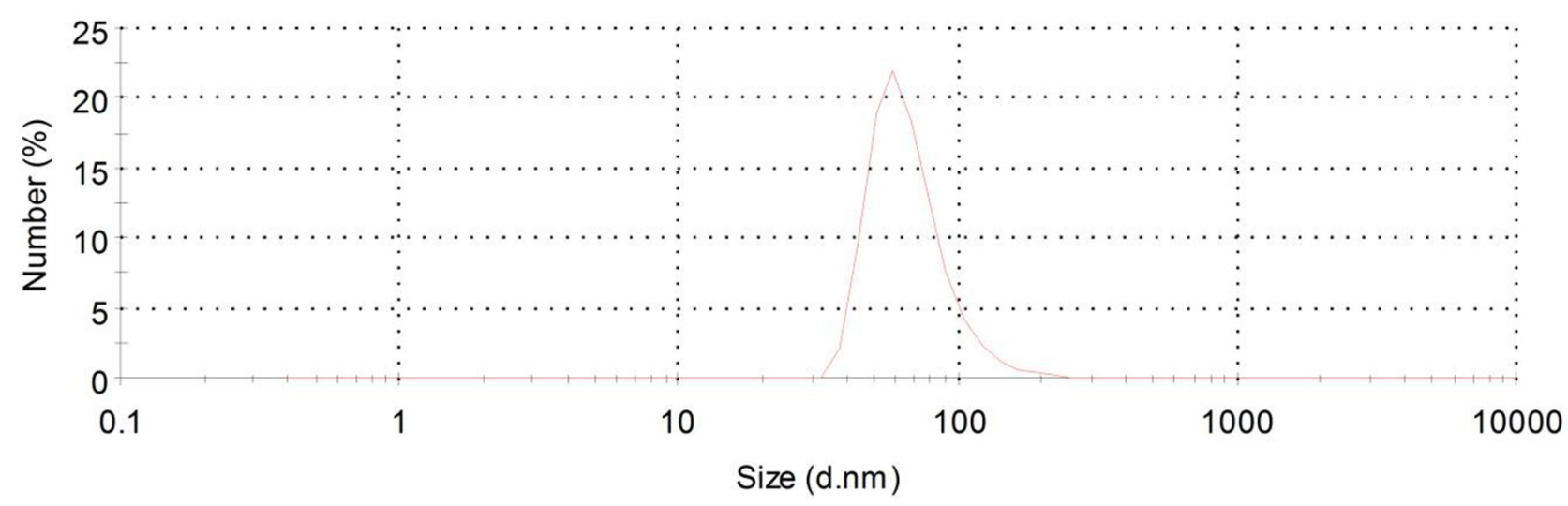

Record 1: PP-18-NH2 pH=5 2:1 1

Figure 2: Size and Zeta potential of DSPE-PEG nanoparticles. 
the IRI+Curcumin and IRI+Cur-NP groups (co-cultured with HK-2 cells), although fluorescence intensity was more significantly reduced in the IRI+Cur-NP group than in the IRI+Curcumin group. This result demonstrated that Cur-NPs can effectively prevent and treat renal IRI by inhibiting oxidative stress response (Figure 6).

\section{Effects of Curcumin and Cur-NP on} malondialdehyde (MDA), superoxide dismutase (SOD), Caspase-3, Bax, and Bcl-2 levels following IRI

Intracellular SOD and MDA contents were determined using the bicinchoninic acid method. MDA level decreased after adding Cur-NPs or non-encapsulated Curcumin. Such reduction was significantly greater in the Cur-NP group than in the Curcumin group. The SOD level increased after adding Cur-NPs or non-encapsulated Curcumin, although such an increase was significantly greater in the Cur-NP group than in the Curcumin group. This result indicated that Curcumin-carrying NPs increased the SOD content and reduced the MDA level (Figure 7).

Expression levels of Caspase-3, Bax, and Bcl2 proteins in each group were detected by Western blot assay. The results showed that addition of Cur-NPs or non-encapsulated Curcumin downregulated the protein expression levels of Caspase-3 and Bax but upregulated Bcl-2 expression. Increase in expression levels was significantly higher in the Cur-NP group than in the Curcumin group (Figure 8).

\section{DISCUSSION}

This study successfully constructed Cur-NPs that display good water solubility and pharmacological features. Our experiments demonstrated that Cur-NPs inhibited the apoptotic rate of HK-2 cells exposed to IRI by reducing reactive oxygen level, by downregulating the expression of pro-apoptotic genes (Caspase-3 and Bax), and by upregulating the expression of anti-apoptotic gene (Bcl-2).

Curcumin possesses anti-microbial [15], antiinflammatory [16], anti-carcinogenic [17], and antioxidant [18] properties. In spite of these properties, the wide use of Curcumin has been limited mainly by its poor water solubility and consequently its poor bioavailability. Intravenous administration of Curcumin, which is a small fat-soluble molecule, is inconvenient and unsafe; moreover, the disadvantages of Curcumin includes its low bioavailability, short half-life, and difficulty in reaching and maintaining therapeutic concentration after entering

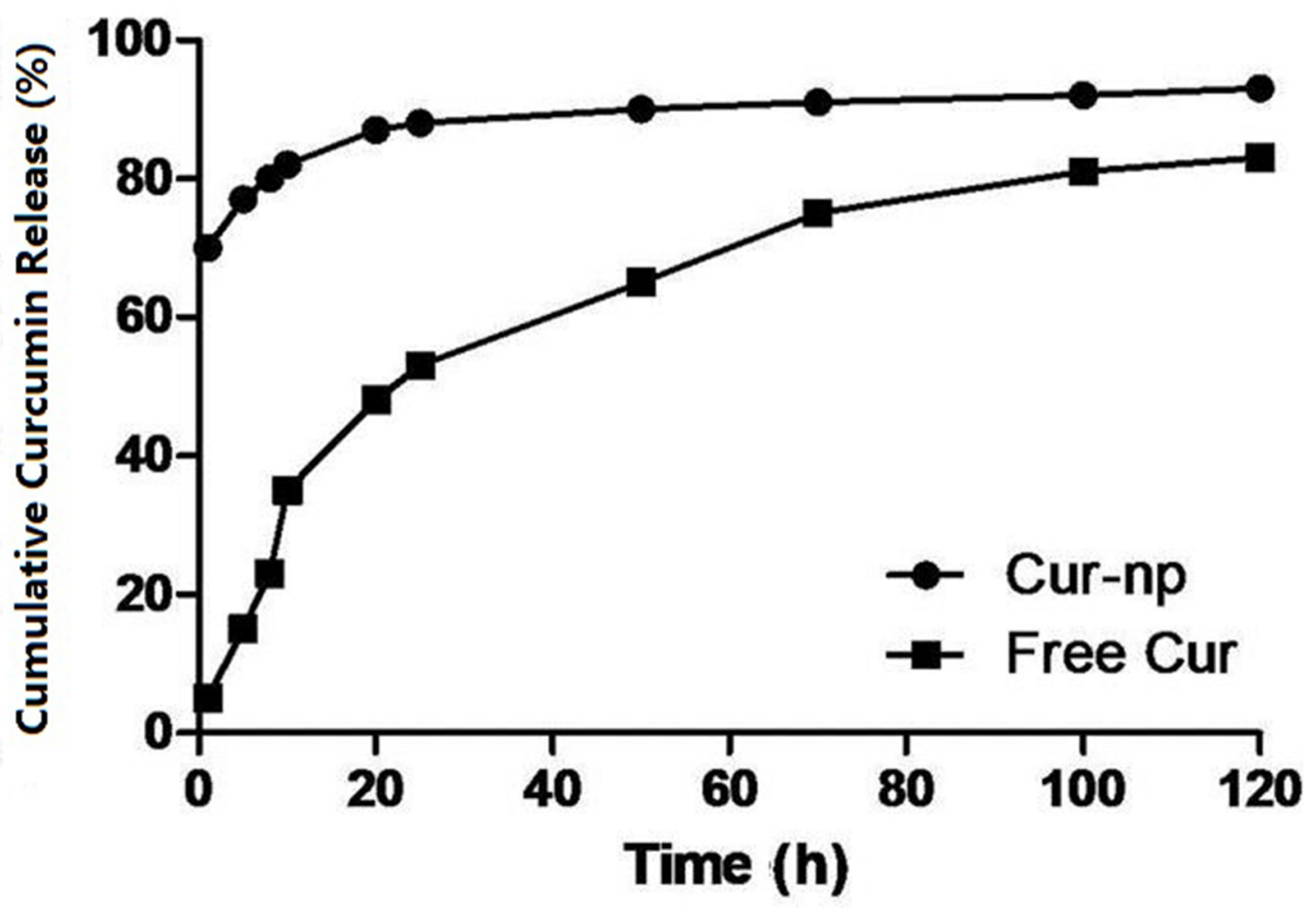

Figure 3: Release curve of Curcumin drug-loading nanoparticles and bare curcumin medicine in vitro. 
the body [19]. Large-dose administration not only fails to significantly increase the in vivo concentration of Curcumin but also induces oxidative stress, generating concentration-dependent toxicity [20]. Thus, developing innovative form of Curcumin to increase its bioavailability and reduce its toxic side effects is extremely necessary. Sasaki [21] described an innovative preparation of Curcumin, in which Curcumin demonstrates improved oral bioavailability and displays the characteristics of nanomaterials. Given the safety, high stability under UV light and high temperature, and excellent water solubility of nanomaterials, they have become increasingly used as effective components in preparing new health care products (beverages, food, supplements, and drugs) [22]. Our data clearly showed that Cur-NPs displayed obviously improved water solubility compared with non-encapsulated Curcumin; moreover, Cur-NPs are approximately $80 \mathrm{~nm}$ in size; thus, they are easily absorbed by the body and that they reach their destination. These results are consistent with those of other studies that have taken advantage of the water solubility of NPs [21, 23]. In addition, investigation on in vitro drug release showed that cumulative release of Cur-NP was significantly higher than that of non-encapsulated Curcumin, indicating that NPs facilitate the release of Curcumin. High cumulative release concentration is obviously essential in increasing the bioavailability of Curcumin. The release behavior may be induced by improved solubility of Curcumin in water, consistent with the findings of Li [24] and Kumar [25].

Although the pathophysiology of IRI is incompletely clear, oxidative stress has been identified as one of the several important mechanisms causing kidney
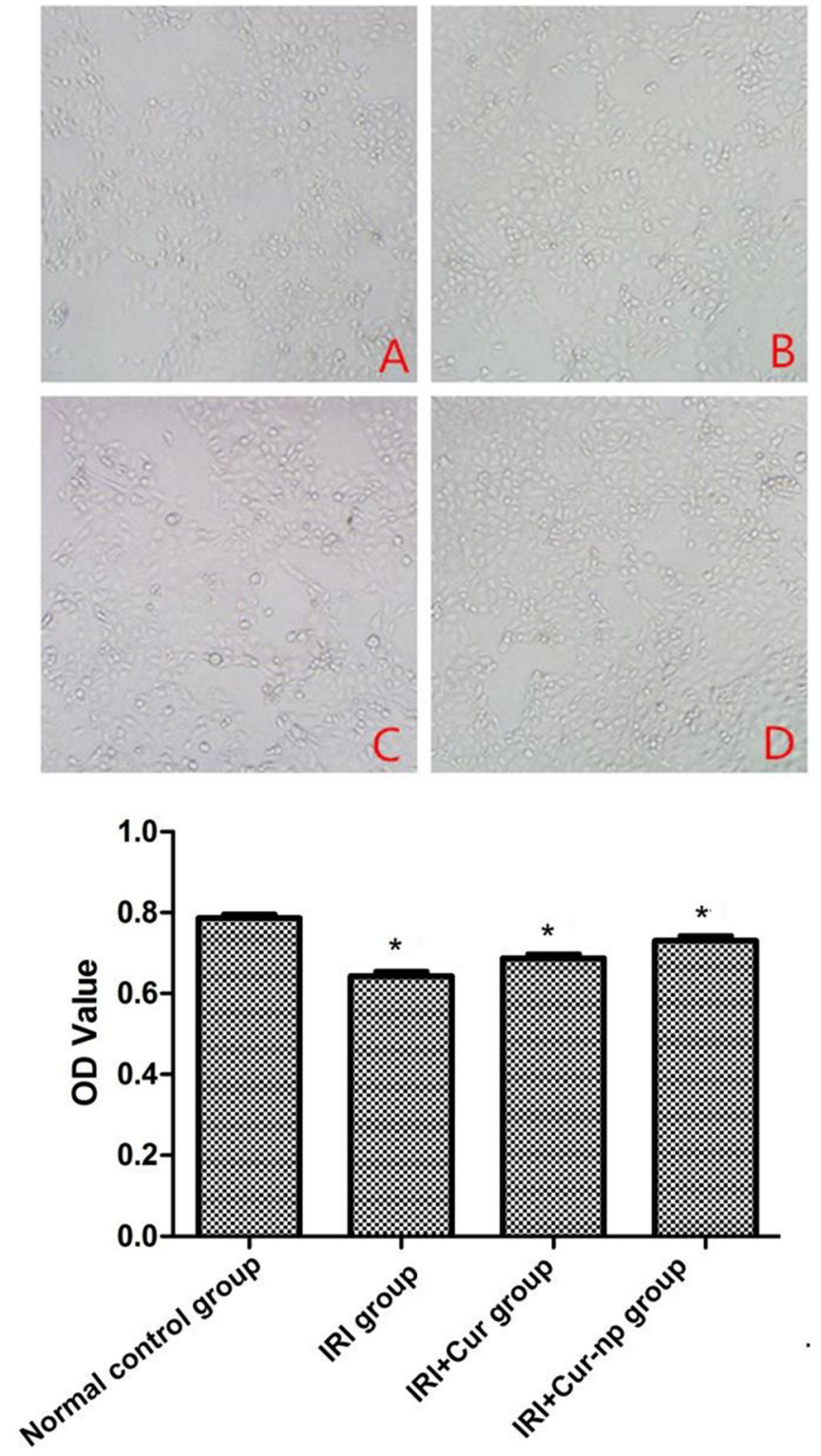

Figure 4: Detection of cell proliferation determined by MTT method. 
failure $[26,27]$. When a kidney experiences ischemia and then reperfusion, excessive ROS generation at the latter phase of ischemia and reperfusion initiates a series of deleterious cellular responses, resulting in inflammation, cell apoptosis, and acute kidney failure [1]. Renal tissues receive abundant blood supply, and the enzymatic and non-enzymatic antioxidant systems are relatively weak [28]. Hence, renal tissues are more sensitive to oxidative stress than the other organs. Rapid elimination of excess ROS during the early stage of ischemia and reperfusion can protect tubular epithelial cells, attenuating the adverse influence of ROS on renal tissues. This study used HK-2 cells to prepare an IRI model. After ischemia-reperfusion (IR), cell viability gradually decreased, whereas the detected ROS level in the cells obviously increased, suggesting that during renal IRI, the number of reactive oxygen molecules and the apoptotic rate significantly increased and that oxidative stress response plays an important role in IRI.
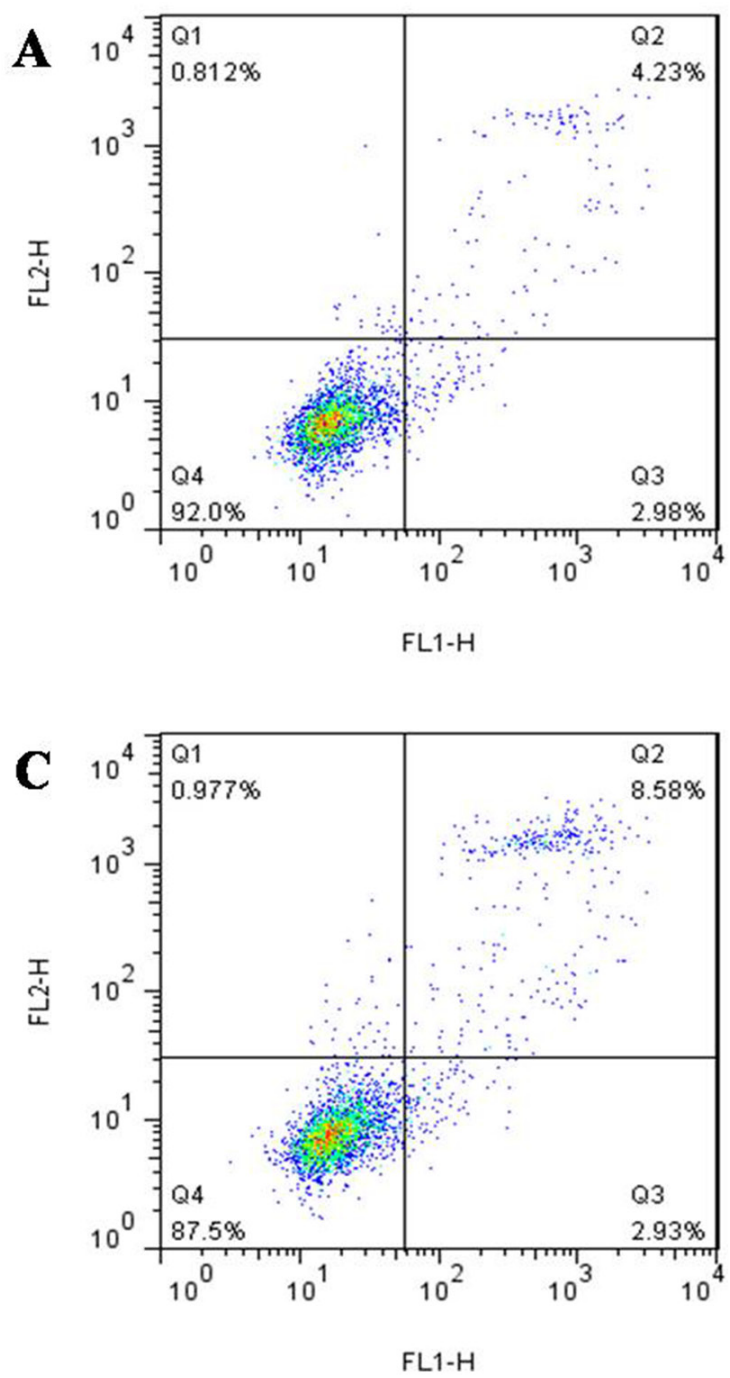

Endogenous substances necessary for ROS elimination include antioxidant enzymes (e.g., SOD, glutathione peroxidase, and catalase) and non-enzymatic antioxidants (e.g., glutathione, uric acid, and vitamins C and E) [29]. Under abnormal states, the capacity to eliminate free radicals from a system is usually impaired. Exogenous antioxidant supplementation is theoretically one of the most effective treatment strategies for IRI $[30,31]$. Studies have shown that antioxidants can be exogenously supplemented through overexpression of antioxidant enzymes by using viral vectors or via direct supplementation with antioxidant enzymes and small molecule antioxidants to treat different IRI types [32-35]. However, overexpression of antioxidant enzymes by using viral vectors is a complex technology and involves problems, such as the concerns on immune responses and slow effects. By contrast, antioxidant enzymes and small-molecule antioxidants, such as vitamin C, cannot achieve the treatment goal given their short half-life in

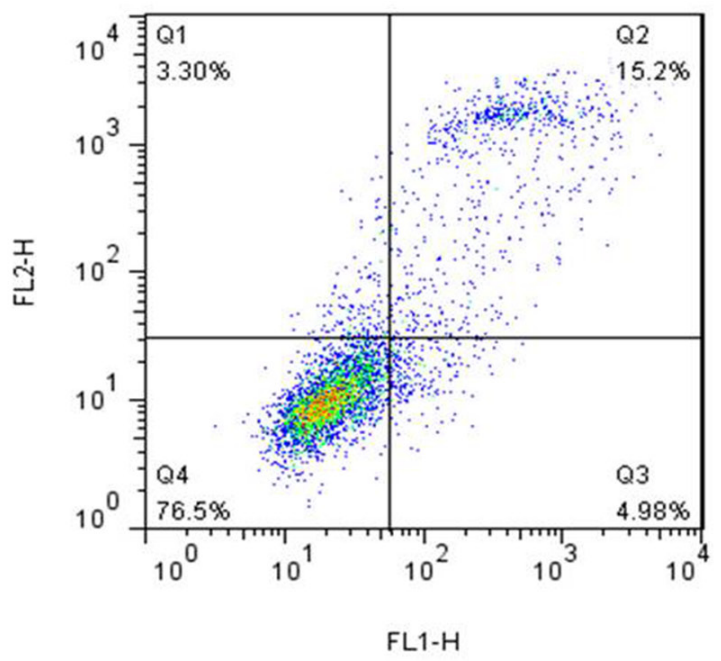

B

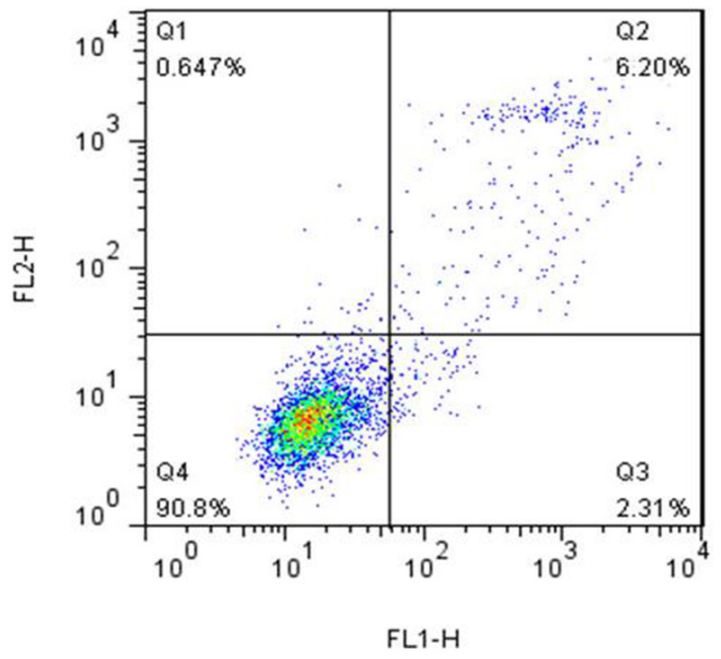

D

Figure 5: The change of apoptosis detected flow cytometry. A. Normal control group. B. IRI group. C. IRI+Cur group. D. IRI+Cur-NPs group. 

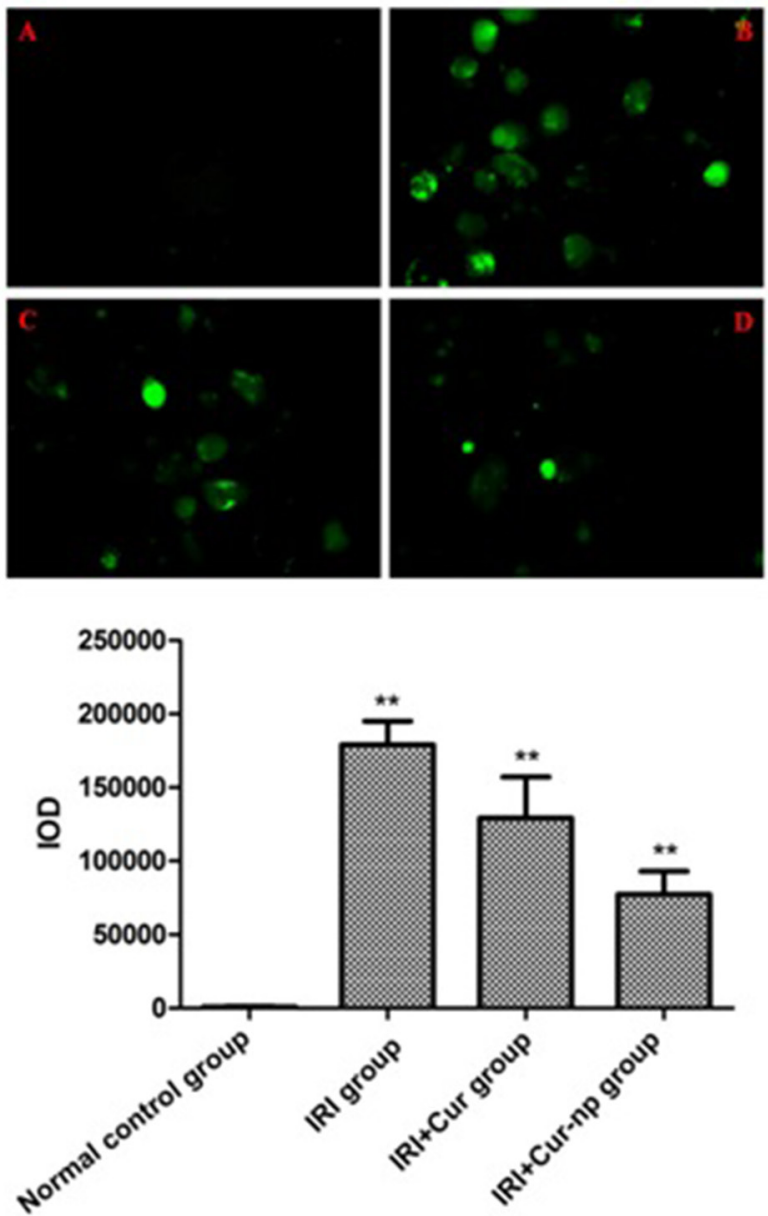

Figure 6: The results of DAPI staining after hypoxia reoxygenation at $24 \mathrm{~h}$. A. Normal control group; B. IRI group; C. IRI+Cur group; D. IRI+Cur-NPs group.

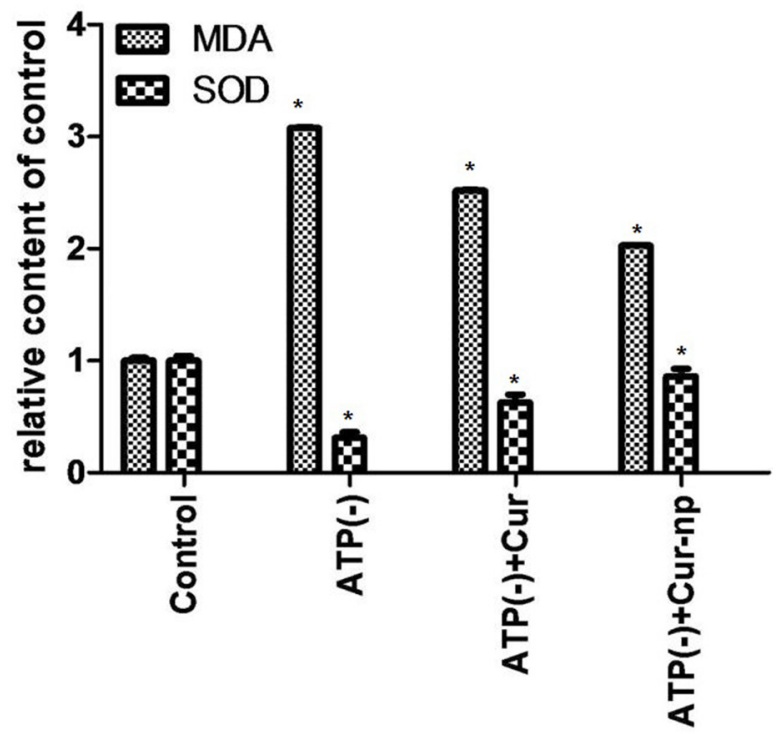

Figure 7: MDA and SOD tests. 
vivo. Curcumin, which displays a powerful antioxidant capability, is a promising candidate as treatment for renal IRI.

Caspase-3 is considered the primary terminal restriction enzyme in renal IRI-induced apoptosis of tubular epithelial cells [35, 36]. Moreover, renal tissue apoptosis is closely related to the expression of the regulatory genes Bcl-2 and Bax [37, 38]. Bcl-2 inhibits apoptosis by inhibiting free radical production, intracellular calcium overload, permeability of mitochondrial membrane, and blockage of Cyt- $\mathrm{C}$ release and caspase activation. Bax is a pro-apoptotic gene that induces apoptosis by promoting cytokine deficiency. Bcl-2 and Bax may form dimers, the ratio of which determines whether a cell survives or becomes apoptotic. MDA is a main product of lipid peroxidation. MDA reflects the degree of damage in tissues and cells caused by lipid peroxidation [39]. In this study, MDA, SOD, apoptosis, caspase-3, Bax, and Bcl-2 proteins were chosen as detection parameters, and Curcumin and Cur-NPs were used as intervention for HK-2 cells exposed to IRI. Compared with the IRI group, the Curcumin and CurNP groups displayed improved cell viability, reduced apoptotic level and MDA content, reduced expression of Caspase-3 protein, downregulated expression of Bax protein, upregulated expression of $\mathrm{Bcl}-2$ protein, and increased level of the antioxidant SOD. These improvements observed in the Cur-NP group were more significant than those in the Curcumin group, indicating that Curcumin can effectively prevent and treat renal IRI by inhibiting oxidative stress response. Furthermore, NPs can significantly promote the inhibitory effect of Curcumin, enhancing the prevention and treatment of renal IRI.

In our study, Cur-NPs demonstrated good water solubility and slowed release, as well as exerted protective effects against oxidative stress in cultured HK-2 cells exposed to renal IRI. Cur-NPs more effectively exerted protective effects than the non-encapsulated Curcumin. Cur-NPs can significantly reduce HK-2 apoptosis and protect the cells by scavenging excessive ROS, by inhibiting the expression levels of Caspase- 3 and Bax protein, and by increasing the expression of Bcl-2. Thus, Cur-NPs may be developed as a promising potential protective agent against renal IRI. Further studies on the effect of Cur-NPs must be conducted.
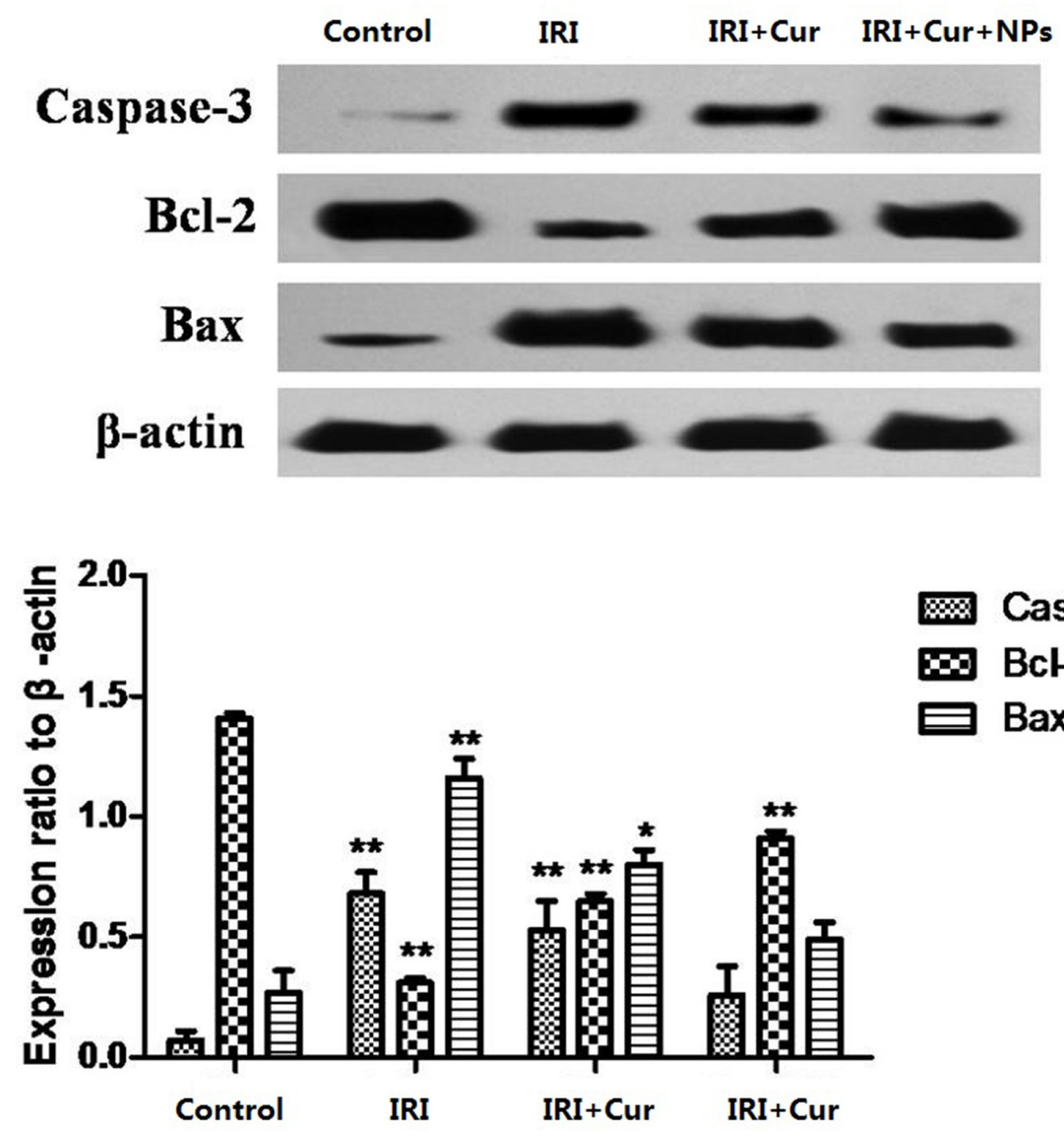

Figure 8: Changes in Expression levels of caspase-3, Bax, and Bcl-2 proteins in each group. 


\section{MATERIALS AND METHODS}

\section{Reagents and instruments}

Curcumin $\left(\mathrm{C}_{21} \mathrm{H}_{20} \mathrm{O}_{6} ;>98 \%\right.$ pure $)$ was purchased from Shaanxi Sciphar Biotechnology Co., Ltd. DSPE and PEG were purchased from Sigma Inc. Apoptosis detection kit (Annexin VFITC/PI), ROS detection kit, protein extraction kit, MDA kit, and SOD kit were all purchased from Nanjing Key Gen Biotech. Co., Ltd.

The following instruments were used: $\mathrm{CO}_{2}$ incubator (Thermo311, USA), $\mathrm{CO}_{2}$ tri-gas incubator (Thermo3131, USA), microplate reader (ELx800, BioTek, USA), flow cytometer (FACSCalibur, Becton-Dickinson, USA), inverted fluorescence microscope (IX51, OLYMPUS, Japan), Western electrophoresis apparatus (164-5051, Bio-Rad, USA), spectrophotometer (UV-2540, Shimadzu, Japan), and gel imager (Gel Doc XR, Bio-Rad, USA).

\section{Cell culture}

The human renal tubular epithelial cell line HK-2 was provided by Nanjing Key Gen Biotech. Co., Ltd. and cultured in a DMEM/F12 culture solution containing 10\% fetal bovine serum (FBS), $100 \mathrm{U} / \mathrm{mL}$ penicillin, and 100 $\mathrm{mg} / \mathrm{L}$ streptomycin. The cells were cultured at $37^{\circ} \mathrm{C}$ under $5 \% \mathrm{CO}_{2}$ saturated humidity. Cells in the logarithmic phase were chosen for the experiments.

\section{Construction of Cur-DSPE-PEG NPs (Cur-NPs)}

Cur-NPs were constructed as previously reported. Briefly, an amount of Res and $20 \mathrm{mg}$ of PVP-b-PCL diblock copolymer were dissolved in $0.4 \mathrm{~mL}$ of acetone. Under moderate stirring at $25^{\circ} \mathrm{C}$, the solution above was added dropwise into $5 \mathrm{~mL}$ of distilled water. The resulting solution was filtered through a $0.22 \mu \mathrm{m}$ filter membrane to remove the unincorporated copolymer aggregates and drugs. The solution was subsequently placed into a dialysis bag (MWCO 12000) and then immersed in redistilled water to thoroughly remove the acetone. Finally, the solution was lyophilized for further use. Empty NPs were prepared in a similar manner but omitting the drug. Green fluorescent coumarin- 6 was incorporated into the NPs to visualize the uptake of polymeric micelles by cells. The lyophilized NPs were dissolved in PBS before use.

\section{In vitro investigation on drug release}

Curcumin release was investigated in vitro by using a dialysis bag. Lyophilized Cur-NPs (10 mg) was suspended in $1 \mathrm{~mL}$ of physiological saline. Equal amounts of Cur-NPs solution and non-encapsulated Curcumin were placed into a dialysis bag (molecular weight cut-off of $12,000)$, which was completely immersed in $0.01 \mathrm{~mol} / \mathrm{L}$ phosphate-buffered saline (PBS, $\mathrm{pH}=7.4$ ). The entire release experiment was performed at $37^{\circ} \mathrm{C}$. At regular intervals, extracellular fluid was removed and an equal amount of new PBS was added. Curcumin content in the outer liquid was measured at different time points through high performance liquid chromatography.

\section{Setting of IRI conditions}

Cells were washed two to three times with PBS, and complete medium containing $10 \%$ FBS was added. The cells were subsequently placed in a hypoxia device $(<1 \%$ oxygen content). Aeration proceeded for $0.5 \mathrm{~h}$, and then the device was sealed. The cells were continuously cultured for $24 \mathrm{~h}$ under ischemia and hypoxia, and then the culture medium was replaced with the same type and volume of medium on which the cells were cultured for another $24 \mathrm{~h}$.

\section{Examination of cell proliferation using the MTT method}

HK-2 cells in the logarithmic phase were diluted to a density of $5 \times 10^{4}$ cells $/ \mathrm{mL}$ by using DMEM/F12 containing $10 \%$ FBS and then inoculated in a 96-well culture plate; each well contains $100 \mu \mathrm{L}$ of medium. The experiment included a normal control group, IR group, IR+Curcumin (IR-Cur) group $(50 \mu \mathrm{M})$, and IR+Curcarrying NP (IR-Cur-NP) group $(50 \mu \mathrm{M})$. Different drug concentrations were separately added into the wells and then the plate was incubated at $37^{\circ} \mathrm{C}$ under $5 \% \mathrm{CO}_{2}$ for 24 h. After incubation, $20 \mu \mathrm{L}$ of MTT $(5 \mathrm{mg} / \mathrm{mL})$ was added into each well, and the plate was continuously incubated at $37^{\circ} \mathrm{C}$ for another $4 \mathrm{~h}$. The supernatant was discarded and $150 \mu \mathrm{L}$ of dimethylsulfoxide was added into each well. The plate was mildly shaken in a shaker for $10 \mathrm{~min}$ to adequately dissolve the crystals. OD was measured using an automatic microplate reader (wavelength, $490 \mathrm{~nm}$ ), and cell inhibition rates under different drug concentrations were calculated by the following formula: Cell inhibition rate $=(\mathrm{OD}$ value of the control group $-\mathrm{OD}$ value of the experimental group/OD value of the control group) $\times$ $100 \%$. Five parallel control wells were used in each group.

\section{Apoptosis detection through flow cytometry}

Cells in the logarithmic phase were digested and then inoculated on a six-well plate. Once the cells adhered onto the wall the following day, the corresponding drugcontaining culture media were added into the cells of the different groups. At the same time, a negative control group was set. After $24 \mathrm{~h}$ of drug action, $0.25 \%$ trypsin (EDTA-free) was used to digest the collected cells, which were subsequently washed twice with PBS (centrifuged at 2,000 rpm for $5 \mathrm{~min}$ ) to collect $5 \times 10^{5}$ cells. A binding buffer $(500 \mu \mathrm{L})$ was added to suspend the cells. After the cell suspension was mixed thoroughly with $5 \mu \mathrm{L}$ of Annexin V-FITC, $5 \mu \mathrm{L}$ of propidium iodide was added 
and mixed thoroughly into the mixture. The system was allowed to react in the dark for 5-15 min at room temperature. The apoptotic conditions were subsequently examined via flow cytometry.

\section{Observation of changes in reactive oxygen content in cells through fluorescence microscopy}

Cells were washed once with PBS and then centrifuged at 2,000 rpm for $5 \mathrm{~min}$. The cells were subsequently collected, and cell concentration was adjusted to $1 \times 10^{6}$ cells $/ \mathrm{mL}$. 2', 7'-Dichlorofluorescin diacetate (DCFH-DA) was diluted with a serum-free culture solution at 1:1,000 to reach a final concentration of $10 \mu \mathrm{M}$. The cells were collected, suspended in prediluted DCFH-DA, and then incubated at $37^{\circ} \mathrm{C}$ for $20 \mathrm{~min}$. The cell culture was turned upside down every 3-5 min to mix the cells and ensure adequate contact between the probes and the cells. The cells were subsequently washed with a serum-free cell culture solution for three times to completely eliminate the DCFH-DA that did not penetrate into the cells. Fluorescence intensity was determined and photographs were obtained via fluorescence microscopy.

\section{Determination of SOD and MDA contents}

After cessation of drug action on the cells, the culture medium was sucked out. The cells were washed three times with a post-nuclear supernatant, digested with $0.25 \%$ trypsin, and then the cell suspension was collected. SOD and MDA contents were determined through spectrophotometry according to the instruction manual.

\section{Detection of protein expression through western blot assay}

After cessation of drug action on the cells, the cells were digested with trypsin and then washed twice with cold PBS. Radioimmunoprecipitation assay lysis buffer $(200 \mu \mathrm{L})$ was added for complete cell lysis. The mixture was thoroughly mixed and then placed in an ice bath for $30 \mathrm{~min}$, during which the mixture was mixed on a vortex mixer for $10 \mathrm{~s}$ every $5 \mathrm{~min}$ to achieve complete lysis. The mixture was subsequently centrifuged at $12,000 \mathrm{rpm}$ for $5 \mathrm{~min}$ for protein extraction, and protein concentration was determined on a spectrophotometer by using the Coomassie brilliant blue G250 method followed by $12 \%$ sodium dodecyl sulfate-polyacrylamide gel electrophoresis electrophoretic separation. The protein was electrotransfered onto a polyvinylidenefluoride membrane, blocked by using 5\% skim milk powder for 1 $\mathrm{h}$ followed by blocking with a primary antibody (1:500) at $4{ }^{\circ} \mathrm{C}$ overnight, and then incubated with secondary antibody (horseradish peroxidase-labeled goat anti-rabbit or goat anti-rat antibody $1: 1,000)$ for $2 \mathrm{~h}$. The results were obtained using the chemiluminescence method, and photographs were captured using a gel image analysis system.

\section{Statistical analyses}

Data are presented as mean \pm standard error. Comparisons were made by using one-way ANOVA followed by multiple comparison (Fisher's least significant difference test), which is available in the SPSS13.0 statistical software (SPSS, Chicago, Illinois, USA). A $p$ value of $<0.05$ indicated statistical significance.

\section{ACKNOWLEDGMENTS}

This study was supported by the National Natural Science Fund of China (No. 81270817).

\section{CONFLICTS OF INTEREST}

The authors declare no conflicts of interest.

\section{REFERENCES}

1. Malek M, Nematbakhsh M. Renal ischemia/reperfusion injury; from pathophysiology to treatment. J Renal Inj Prev. 2015; 4:20-27.

2. Moreira MA, Irigoyen MC, Saad KR, Saad PF, Koike MK, Montero EF, Martins JL. N-acetylcysteine reduces the renal oxidative stress and apoptosis induced by hemorrhagic shock. J Surg Res. 2016; 203:113-120.

3. Yang Z, Zhong Z, Li M, Xiong Y, Wang Y, Peng G, Ye Q. Hypothermic machine perfusion increases A20 expression which protects renal cells against ischemia/reperfusion injury by suppressing inflammation, apoptosis and necroptosis. Int J Mol Med. 2016; 38:161-171.

4. Zahedi K, Wang Z, Barone S, Prada AE, Kelly CN, Casero RA, Yokota N, Porter CW, Rabb H, Soleimani M. Expression of SSAT, a novel biomarker of tubular cell damage, increases in kidney ischemia-reperfusion injury. Am J Physiol Renal Physiol. 2003; 284:F1046-1055.

5. Zou YR, Zhang J, Wang J, Peng L, Li GS, Wang L. Erythropoietin Receptor Activation Protects the Kidney From Ischemia/Reperfusion-Induced Apoptosis by Activating ERK/p53 Signal Pathway. Transplant Proc. 2016; 48:217-221.

6. Salvadori M, Rosso G, Bertoni E. Update on ischemiareperfusion injury in kidney transplantation: Pathogenesis and treatment. World J Transplant. 2015; 5:52-67.

7. Akyuz S, Turan F, Gurbuzler L, Arici A, Sogut E, Ozkan O. The Anti-Inflammatory and Antioxidant Effects of Curcumin in Middle Ear Infection. J Craniofac Surg. 2016; 27:e494-497.

8. Tvrda E, Tusimova E, Kovacik A, Paal D, Greifova H, Abdramanov A, Lukac N. Curcumin has protective and 
antioxidant properties on bull spermatozoa subjected to induced oxidative stress. Anim Reprod Sci. 2016.

9. Farombi EO, Ekor M. Curcumin attenuates gentamicininduced renal oxidative damage in rats. Food Chem Toxicol. 2006; 44:1443-1448.

10. Mou X, Ali Z, Li S, He N. Applications of Magnetic Nanoparticles in Targeted Drug Delivery System. J Nanosci Nanotechnol. 2015; 15:54-62.

11. Sahoo N, Sahoo RK, Biswas N, Guha A, Kuotsu K. Recent advancement of gelatin nanoparticles in drug and vaccine delivery. Int J Biol Macromol. 2015; 81:317-331.

12. Li X, Zhen D, Lu X, Xu H, Shao Y, Xue Q, Hu Y, Liu B, Sun W. Enhanced cytotoxicity and activation of ROSdependent c-Jun NH2-terminal kinase and caspase-3 by low doses of tetrandrine-loaded nanoparticles in Lovo cells--a possible Trojan strategy against cancer. Eur J Pharm Biopharm. 2010; 75:334-340.

13. Kim J, Lee YM, Kim H, Park D, Kim J, Kim WJ. Phenylboronic acid-sugar grafted polymer architecture as a dual stimuli-responsive gene carrier for targeted antiangiogenic tumor therapy. Biomaterials. 2016; 75:102-111.

14. Lestari ML, Muller RH, Moschwitzer JP. Systematic screening of different surface modifiers for the production of physically stable nanosuspensions. J Pharm Sci. 2015; 104:1128-1140.

15. Muglikar S, Patil KC, Shivswami S, Hegde R. Efficacy of curcumin in the treatment of chronic gingivitis: a pilot study. Oral Health Prev Dent. 2013; 11:81-86.

16. Wang J, Kang YX, Pan W, Lei W, Feng B, Wang XJ. Enhancement of Anti-Inflammatory Activity of Curcumin Using Phosphatidylserine-Containing Nanoparticles in Cultured Macrophages. Int J Mol Sci. 2016; 17.

17. Norris L, Karmokar A, Howells L, Steward WP, Gescher A, Brown K. The role of cancer stem cells in the anticarcinogenicity of curcumin. Molecular nutrition \& food research. 2013; 57:1630-1637.

18. Wang D, Hu J, Lv L, Xia X, Liu J, Li X. Enhanced inhibitory effect of curcumin via reactive oxygen species generation in human nasopharyngeal carcinoma cells following purple-light irradiation. Oncol Lett. 2013; 6:81-85.

19. Storka A, Vcelar B, Klickovic U, Gouya G, Weisshaar S, Aschauer S, Bolger G, Helson L, Wolzt M. Safety, tolerability and pharmacokinetics of liposomal curcumin in healthy humans. Int J Clin Pharmacol Ther. 2015; 53:54-65.

20. Tian B, Wang Z, Zhao Y, Wang D, Li Y, Ma L, Li X, Li J, Xiao N, Tian J, Rodriguez R. Effects of curcumin on bladder cancer cells and development of urothelial tumors in a rat bladder carcinogenesis model. Cancer Lett. 2008; 264:299-308.

21. Sasaki H, Sunagawa Y, Takahashi K, Imaizumi A, Fukuda H, Hashimoto T, Wada H, Katanasaka Y, Kakeya H, Fujita $\mathrm{M}$, Hasegawa $\mathrm{K}$, Morimoto T. Innovative preparation of curcumin for improved oral bioavailability. Biol Pharm Bull. 2011; 34:660-665.
22. Parakhonskiy BV, Yashchenok AM, Konrad M, Skirtach AG. Colloidal micro- and nano-particles as templates for polyelectrolyte multilayer capsules. Adv Colloid Interface Sci. 2014; 207:253-264.

23. Bollimpelli VS, Kumar P, Kumari S, Kondapi AK. Neuroprotective effect of curcumin-loaded lactoferrin nano particles against rotenone induced neurotoxicity. Neurochem Int. 2016; 95:37-45.

24. Li X, Yuan H, Zhang C, Chen W, Cheng W, Chen X, Ye $\mathrm{X}$. Preparation and in-vitro/in-vivo evaluation of curcumin nanosuspension with solubility enhancement. J Pharm Pharmacol. 2016; 68:980-988.

25. Kumar A, Ahuja A, Ali J, Baboota S. Curcumin loaded nano globules for solubility enhancement: preparation, characterization and ex vivo release study. J Nanosci Nanotechnol. 2012; 12:8293-8302.

26. Qiao X, Li RS, Li H, Zhu GZ, Huang XG, Shao S, Bai B. Intermedin protects against renal ischemia-reperfusion injury by inhibition of oxidative stress. Am J Physiol Renal Physiol. 2013; 304:F112-119.

27. Huang Q, Wang Q, Zhang S, Jiang S, Zhao L, Yu L, Hultstrom M, Patzak A, Li L, Wilcox CS, Lai EY. Increased hydrogen peroxide impairs angiotensin II contractions of afferent arterioles in mice after renal ischaemia-reperfusion injury. Acta Physiol (Oxf). 2016.

28. Bharti VK, Srivastava RS, Kumar H, Bag S, Majumdar AC, Singh G, Pandi-Perumal SR, Brown GM. Effects of melatonin and epiphyseal proteins on fluoride-induced adverse changes in antioxidant status of heart, liver, and kidney of rats. Adv Pharmacol Sci. 2014; 2014:532969.

29. Kim MJ, Woo SW, Kim MS, Park JE, Hwang JK. Antiphotoaging effect of aaptamine in UVB-irradiated human dermal fibroblasts and epidermal keratinocytes. J Asian Nat Prod Res. 2014; 16:1139-1147.

30. Bi W, Wang Y, Sun G, Zhang X, Wei Y, Li L, Wang X. Paclitaxel-resistant HeLa cells have up-regulated levels of reactive oxygen species and increased expression of taxol resistance gene 1. Pak J Pharm Sci. 2014; 27:871-878.

31. Siriussawakul A, Zaky A, Lang JD. Role of nitric oxide in hepatic ischemia-reperfusion injury. World J Gastroenterol. 2010; 16:6079-6086.

32. Kirkby K, Baylis C, Agarwal A, Croker B, Archer L, Adin C. Intravenous bilirubin provides incomplete protection against renal ischemia-reperfusion injury in vivo. Am J Physiol Renal Physiol. 2007; 292:F888-894.

33. Masuda Y, Vaziri ND, Takasu C, Li S, Robles L, Pham C, Le A, Vo K, Farzaneh SH, Stamos MJ, Ichii H. Salutary effect of pre-treatment with an Nrf2 inducer on ischemia reperfusion injury in the rat liver. Gastroenterol Hepatol (Que). 2014; 1:1-7.

34. Al-Maghrebi M, Renno WM, Al-Ajmi N. Epigallocatechin3-gallate inhibits apoptosis and protects testicular seminiferous tubules from ischemia/reperfusion-induced inflammation. Biochem Biophys Res Commun. 2012; 420:434-439. 
35. Ulbrich F, Schallner N, Coburn M, Loop T, Lagreze WA, Biermann J, Goebel U. Argon inhalation attenuates retinal apoptosis after ischemia/reperfusion injury in a timeand dose-dependent manner in rats. PLoS One. 2014; 9:e115984.

36. Yamada M, Kaibori M, Tanaka H, Habara K, Hijikawa T, Tanaka Y, Oishi M, Okumura T, Nishizawa M, Kwon AH. alpha-lipoic acid prevents the induction of iNOS gene expression through destabilization of its mRNA in proinflammatory cytokine-stimulated hepatocytes. Dig Dis Sci. 2012; 57:943-951.

37. Xu T, Wu X, Chen Q, Zhu S, Liu Y, Pan D, Chen X, $\mathrm{Li}$ D. The anti-apoptotic and cardioprotective effects of salvianolic acid a on rat cardiomyocytes following ischemia/reperfusion by DUSP-mediated regulation of the ERK1/2/JNK pathway. PLoS One. 2014; 9:e102292.

38. Xiao ZY, Banan B, Jia J, Manning PT, Hiebsch RR, Gunasekaran M, Upadhya GA, Frazier WA, Mohanakumar T, Lin Y, Chapman WC. CD47 blockade reduces ischemia/ reperfusion injury and improves survival in a rat liver transplantation model. Liver Transpl. 2015; 21:468-477.

39. Granci V, Cai F, Lecumberri E, Clerc A, Dupertuis YM, Pichard C. Colon cancer cell chemosensitisation by fish oil emulsion involves apoptotic mitochondria pathway. Br J Nutr. 2013; 109:1188-1195. 